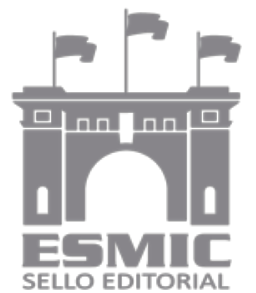

\author{
Revista Científica General José María Córdova \\ ISSN 1900-6586 (impreso), 2500-7645 (en línea) \\ Volumen 16, Número 21, enero-marzo 2018, pp. 83-99 \\ http://dx.doi.org/10.21830/19006586.313
}

Citación: Mutis Barreto, C. A. y Otálora-Buitrago, A. (2018, enero-marzo). La promoción del liderazgo rural para la paz a partir del aprendizaje experiencial. Rev. Cient. Gen. José Maria Córdova, 16 (21), 83-99. DOI: http://dx.doi.org/10.21830/19006586.313

\title{
La promoción del liderazgo rural para la paz a partir del aprendizaje experiencial
}

Sección: EdUCACión

Artículo de investigación científica y tecnológica

Claudia Aixa Mutis Barreto ${ }^{a}$

Universidad de La Salle, Bogotá, D. C., Colombia

Adriana Otálora-Buitrago ${ }^{\mathrm{b}}$

Universidad de La Salle, Bogotá, D. C., Colombia

\section{The promotion of rural leadership for peace based on experiential learning}

A promoção da liderança rural para a paz, baseada na aprendizagem expriencial

La promotion du leadership rural pour la paix, fondée sur un apprentissage expérientielle

Recibido: 12 de agosto de 2017 • Aceptado: 28 de octubre de 2017

a https://orcid.org/0000-0001-6950-730X - Contacto: clamutis@unisalle.edu.co

b https://orcid.org/0000-0002-8594-9412 - Contacto: aotalora@unisalle.edu.co 
Resumen. La participación de los jóvenes universitarios lasallistas en actividades complementarias, como la ofertada por los centros de interés o clubes equino, bovino, canino y ovino-caprino, es una oportunidad en la formación de líderes que pueden multiplicar su acción de transformación social cuando se aproximan al sector rural. En este estudio, se realizaron entrevistas a estudiantes líderes de los diferentes clubes y se aplicaron encuestas a estudiantes participantes en eventos que agrupan representantes del sector agropecuario. Se concluye que el aprendizaje experiencial a través de estos centros es un generador de líderes, quienes aportarán al desarrollo rural estableciendo relaciones horizontales e incluyentes en su accionar profesional.

Palabras claves: aprendizaje experiencial; construcción de paz; desarrollo rural; formación política; liderazgo.

\begin{abstract}
The participation of young Lasallian university students in complementary activities, such as those offered by the centers of interest, integrated by members of equine, bovine, canine and sheep-goat clubs, becomes an opportunity to produce leaders who can multiply their action of social transformation in their approaches to the rural sector. In this study, interviews were conducted with leading students of the different clubs, as well as surveys to students participating in events that assemble representatives of the agricultural sector. It is concluded that experiential learning through these centers is a generator of leaders who will contribute to rural development through the establishment of horizontal and inclusive relationships in their professional actions.
\end{abstract}

Keywords: experiential learning; peace building; political formation; rural development.

Resumo. A participação de jovens universitários Lasallistas em atividades complementares, tal como as ofertadas pelos centros de interesse ou clubes equino, bovino, canino e ovino-caprino, são uma oportunidade na formação de líderes que podem multiplicar sua ação de transformação social quando aproximam-se ao sector rural. Neste estudo, foram realizadas entrevistas com os melhores estudantes de clubes diferentes, e com base em sondagens realizadas junto dos estudantes que participam em eventos organizados pelo sector agropecuário. Conclui-se que a aprendizagem experiencial através destes centros é um gerador de líderes que contribuem para o desenvolvimento rural, estabelecendo relaçóes horizontais e inclusivas no campo de ação profissional.

Palabras chave: aprendizagem experiencial; construção de paz; desenvolvimento rural; formação política; liderança.

Résumé. La participation de jeunes étudiants universitaires lasalliens à des activités complémentaires, telles que celles offertes par centres d'intérêt ou clubs équins, bovins, canins et ovins-caprins, est une opportunité pour former des leaders capables de multiplier leur action de transformation sociale lorsqu'ils s'approchent au secteur rural. Dans cette étude, des entrevues ont été menées aux meilleurs étudiants de différents clubs et des sondages ont été effectués auprès d'élèves participant à des événements regroupant des représentants du secteur agricole. Il est conclu que l'apprentissage expérientiel à travers ces centres est un générateur de leaders, qui contribueront au développement rural en établissant des relations horizontales et inclusives dans leur action professionnelle.

Mots-clés: apprentissage expérientiel ; construction de la paix ; développement rural ; formation politique ; leadership. 


\section{Introducción}

Uno de los grandes retos que enfrenta la universidad hoy en día es formar a sus jóvenes estudiantes como actores capaces de generar un impacto en la sociedad y de transformar desde su ejercicio profesional aquellas situaciones injustas que limitan el buen vivir de sus conciudadanos. En apariencia, es en el ámbito de las ciencias sociales que debería promoverse la toma de conciencia y el liderazgo político de los jóvenes profesionales con miras a la transformación social. Sin embargo, la escisión que se ha establecido entre ciencias sociales, humanas, exactas y de la salud es en buena parte culpable de la débil conciencia que existe en ellas sobre el papel que de facto desempeñan en la construcción social.

En el marco de los recientes avances en construcción de paz, las preguntas que se elevan desde el Estado, la academia y la sociedad civil en torno al buen vivir cobran especial relevancia, en particular, dada la necesidad de que cada uno de estos actores asuma su parte de responsabilidad en la consecución de una sociedad incluyente y en la que todos sus integrantes gocen de condiciones de vida digna y de bienestar.

Tal es el caso de las ciencias agropecuarias, las cuales se aproximan más a la lógica de las ciencias exactas y de las ciencias de la salud, y, sin embargo, es a partir de estas mismas áreas de conocimiento que se posibilita una aproximación certera y expedita de la universidad al mundo rural.

La formación política para estos jóvenes, por tanto, no es explícita, y en ocasiones tiende a considerarse como accesoria a los conocimientos propios de su área de conocimiento. Aun así, son los jóvenes médicos veterinarios, zootecnistas y administradores de empresas agropecuarias, que deciden desarrollar su ejercicio profesional en el sector rural, quienes tienen una posibilidad directa y clara de establecer interrelaciones con los actores rurales y, por tanto, quienes tienen la responsabilidad de incidir políticamente en tales zonas.

Entiéndase la incidencia política como la capacidad de formar interrelaciones democráticas y respetuosas a partir de la igual dignidad humana con los distintos actores del sector rural. Así, un joven profesional de estas áreas actúa como un interlocutor que desde el conocimiento científico puede propiciar el manejo adecuado de la enfermedad y la salud animal, de las estrategias productivas o administrativas del campo, o de la generación de mejores oportunidades para sus interlocutores, en lo que sería entendido como un mayor desarrollo o nivel de vida.

Hasta acá no resulta tan extraña la incidencia de estos jóvenes profesionales en la configuración de mejores alternativas para los ciudadanos rurales; con todo, al realizar una aproximación desde el buen vivir al papel político de estos jóvenes, este se ve magnificado por la oportunidad de establecer diálogos interculturales y democráticos incluyentes, en lo que a todas luces contribuye a la construcción de paz en la ruralidad.

Así, la promoción del liderazgo de estos jóvenes en el sector rural se constituye tanto en un reto, como en una obligación de la universidad. En tal sentido, en este artículo se 
expone la experiencia de los centros de interés, club bovino, ovino-caprino, canino y equino, conformados por futuros profesionales lasallistas del área de las agrociencias. Con tal propósito, se realizó una encuesta a estos jóvenes, y se entrevistó a los líderes de los clubes bovino, equino y ovino-caprino.

\section{El ejercicio profesional en el sector rural como un escenario de liderazgo democrático}

Si bien no todos los profesionales del área de las agrociencias tienen la oportunidad de ejercer como profesionales en el sector rural, se asume que quienes lo hagan, tendrán un papel de influencia allí, toda vez que a partir del conocimiento científico de asistencia técnica en temas de salud y producción animal es posible que forjen vínculos a través de las cuales pueden promover las relaciones democráticas y contradecir así los paradigmas modernos del desarrollo, los cuales, al ser homogeneizantes, resultan excluyentes frente a visiones alternativas de mundo, propias de cosmovisiones como las de las comunidades rurales.

La interacción entre distintas comunidades, las cuales forman parte de la construcción social de la realidad y quienes tradicionalmente han sido excluidas de los escenarios del desarrollo moderno, constituye un punto de partida en la comprensión de lo que significa ser un ciudadano rural, y como respuesta a las desventajas que el actual modelo civilizatorio le ha impuesto a las comunidades rurales. Así, la interacción forma parte de una comprensión integradora del mundo, en términos de lo que desde el buen vivir se entiende como una respuesta "ante la desarmonía ocasionada por diversas acciones humanas, partiendo de las consecuencias del actual modelo de desarrollo, como una respuesta estructural civilizatoria para hacer una cultura de justicia socio ambiental" (Burgos-Ayala, 2016, p. 201).

Caudillo-Félix (2012) realiza una interesante reflexión en torno a la construcción del buen vivir como un diálogo intercultural en el cual se propicia la transformación mutua entre principios propios de las comunidades indígenas y los intereses de otros actores, fundamentalmente académicos europeos, quienes fortalecen la emergencia de fuerzas opositoras al capitalismo y a las políticas neoliberales. En tal contexto,

[1] a ciudad se constituyó como un espacio de concentración de poder y riquezas, donde no se daba cabida a lo rural. Así, la urbe se apropia del espacio y de los recursos naturales en aras de la modernidad y el progreso. Por ello se justifica la dominación y el despojo capitalista a costa de la ruina y el saqueo del campesino, de la pérdida de identidades indígenas y afrodescendientes. Por lo que la producción del modelo urbano significa la imposición de condiciones de dominio y violencia para someter a las formas rurales. (Rodríguez, 2014, p. 200) 
Debe señalarse que si bien la educación política no constituye un eje en la formación disciplinar de estos profesionales, la educación de profesionales con un fuerte espíritu de servicio y contribución a la sociedad sí es parte del Enfoque Formativo Lasallista (EFL). En esa medida, cobra relevancia la inclusión de otras comunidades, como lo son las comunidades rurales, en este tipo de diálogos interculturales; en otras palabras, el buen vivir no puede limitarse a las comunidades indígenas ni a los grandes centros de poder, sino que debería involucrar a diversas comunidades, entre estas, a las comunidades rurales;

esta tradición insta desde la formación a que cada persona cultive su sensibilidad social, su responsabilidad tanto personal como profesional, y su compromiso con la justicia social, dentro de la óptica de una opción preferencial por los empobrecidos y por todos los seres humanos que viven en las fronteras de la deshumanización. (Universidad de La Salle, 2008, p. 14)

Se debe destacar cómo la justicia social y la responsabilidad profesional son maneras de volver sobre lo humano y de rescatar la humanidad. Es decir, a partir del compromiso con la justicia social se retorna a lo humano, y como diría Chantal Mouffe (1999), se retorna a lo político. La opción preferencial por los empobrecidos implica el reconocimiento de la injusticia social y la búsqueda de mecanismos para transformar tal realidad, para incluir a los excluidos y para construir nuevos escenarios de convivencia y bienestar social.

Así, el buen vivir "no se trata simplemente de hacer mejor lo realizado hasta ahora. Como parte de la construcción colectiva de un nuevo pacto de convivencia social y ambiental es necesario construir nuevos espacios de libertad y romper todos los cercos que impiden su vigencia" (Acosta, 2010, p. 26); buena parte de estos espacios se encuentra en la relación entre ciudadanos urbanos y rurales, así como en la existencia de líderes y actores sociales dispuestos a entablar diálogos interculturales y a promover nuevas formas de construcción colectiva.

Ahora bien, esto implica preguntarse por la caracterización de las comunidades rurales, especialmente en el marco de la dicotomía urbano-rural, que ha marcado una relación de subordinación de lo rural frente a lo urbano y que ha dejado en franca desventaja a los primeros. En tal sentido, Rodríguez (2014) señala que

[1]a construcción actual de alternativas al desarrollo en la relación campo-ciudad se da en el contexto de una crisis del modelo urbano industrial capitalista, que hace necesario replantearse la viabilidad futura de las ciudades. Este contexto de crisis ha agudizado las desigualdades económicas, sociales y territoriales en el medio rural, con la implantación asimétrica de relaciones de poder y con una participación desigual de los campesinos en los espacios de decisión y de elaboración de políticas públicas. (p. 213)

Es aquí en donde estos jóvenes se constituyen como promotores del cambio social, bien sea como actores incidentes en las comunidades a través de su ejercicio profesional, o bien como intermediarios entre distintos actores, como pueden ser las comunidades 
de productores campesinos agrícolas o pecuarios, los empresarios y representantes de las asociaciones de empresarios, o el mismo Estado, en la formulación, implementación y evaluación de las políticas públicas que afectan al sector rural.

Si bien no todos los jóvenes que expresan una vocación por estas áreas de conocimiento provienen del sector rural, puede afirmarse que todos aquellos quienes optan por realizar su ejercicio profesional en el campo se constituyen como líderes en la comunidad rural. De esta manera, la promoción de un estilo de liderazgo incluyente, dinámico, proactivo y transformacional en los jóvenes, futuros profesionales del área de las agrociencias, se constituye como una oportunidad en la creación de escenarios de interacción entre los "mundos" urbano y rural, así como en el afianzamiento de liderazgos democráticos en uno y otro sector.

Valga anotar que el tipo de liderazgo ejercido por estos jóvenes (sea autoritario, democrático o laissez faire) tiene efecto además en la forma como sus colaboradores o seguidores comprenden su propio papel frente a los demás actores. Así, el ejercicio de un liderazgo autoritario bien puede reforzar las representaciones de dominación que han perpetuado las relaciones inequitativas existentes entre el campo y la ciudad, al tiempo que el ejercicio de un liderazgo más democrático puede, a manera de efecto secundario, promover el fortalecimiento de las comunidades rurales como actores políticos e interlocutores válidos frente a las asociaciones de empresarios o el mismo Estado.

Esto último forma parte también del EFL, en cuanto

asume, entonces, que la relación educativa va más allá de lo estrictamente funcional, pues propicia la interacción de visiones personales, culturales y creencias diferentes y se convierte así en un laboratorio en el que se promueve la construcción y reconstrucción democrática y ética del tejido social. (Universidad de La Salle, 2008, p. 15)

\section{Los centros de interés: una apuesta por la formación integral}

A partir del año 1983 se propició nuestra unidad de análisis: la conformación de grupos por parte de los estudiantes lasallistas, quienes por interés, afinidad o gusto en una temática particular impulsaron diversas apuestas en formas alternativas de acceder al conocimiento. En el caso de los clubes bovino, ovino-caprino, equino y canino, se buscó que aquellos estudiantes con un interés particular en una especie animal, lograran un conocimiento más profundo a través de la realización de cursos, días de campo, reuniones con gremios y diversas actividades dentro y fuera de la universidad. En tal propósito se vinculó no solamente a estudiantes, sino también a docentes, administrativos, egresados e invitados especiales para enriquecer el grupo.

Estos grupos se denominaron clubes y estaban organizados así: Club Equino, Club Bovino, Club Ovino-Caprino, Club de Apoyo Empresarial Agropecuario, Club Felino, Club de Zoocría y Club de Coleo. Como resultado de las actividades que los estudiantes de la época promovieron, existe evidencia que los profesionales lasallistas que participaron 
en los clubes han sido reconocidos por su liderazgo y son quienes han asumido las presidencias de las diferentes asociaciones del sector pecuario y de mascotas. Los ejemplos más claros se han dado en las presidencias de diferentes asociaciones, como la Asosimmental, Asocriollo, Asociación de Caballistas del Caquetá, entre otras.

Un aspecto que se debe destacar en este sentido es la dinámica que adoptaron tales clubes en el desarrollo de sus capacidades y habilidades profesionales, al encontrar en su motivación un factor generador de éxito. Si bien las actividades en un principio eran lideradas exclusivamente por los integrantes de cada club, en la medida en que los grupos fueron incrementando su participación en eventos, contaron con apoyo institucional. Este espacio pedagógico permitió estructurar de mejor manera la organización, definición de roles y responsabilidades mediante la elección de sus propios presidentes, vicepresidentes, tesoreros y secretarios, en algunos casos incluso con la adopción de estatutos.

En términos de la organización del club mismo, de la conformación de herramientas para su adecuado funcionamiento, del establecimiento de relaciones con el sector productivo y de la motivación a otros estudiantes para propiciar su vinculación a los clubes, se trató de un ejercicio de formación en liderazgo que, como se mencionó antes, vio materializados sus frutos en el desempeño de estos profesionales.

Tal es el caso del Club Canino, el cual logró su vinculación con el Club Canino Colombiano y de esta manera generó lazos que les permitieron a los estudiantes acompañar al Club en sus diferentes exhibiciones nacionales e internacionales; el club tuvo tal impacto en la vida profesional de sus integrantes, que años después algunos egresados se vincularon al Club Canino como criadores e incluso uno de ellos llegó a la presidencia de esta asociación.

A partir del año 2009, los clubes se convirtieron en los Centros de Interés, en los cuales los estudiantes también se agrupaban por motivación personal, con el fin de acercarse y tener más conocimiento sobre alguna especie animal en particular y buscar a través de la vinculación con diferentes asociaciones o gremios un acercamiento al trabajo, manejo y relación con el sector. En esta nueva etapa las agrupaciones deben ser lideradas y conformadas exclusivamente por estudiantes, con el fin de darles más autonomía y de que desarrollen ampliamente su potencial.

De esta manera, los centros de interés materializan

la educación personalizadora que promueve el desarrollo humano de quienes integramos la comunidad universitaria, esto es, una educación que busca en todo momento el respeto de la dignidad de cada quien y el despliegue de todas sus potencialidades, gracias a una adecuada interacción con sus grupos de referencia y con la sociedad. (Universidad de La Salle, 2008, p. 13)

En ese sentido, son un espacio para que sus integrantes desarrollen su vocación profesional desde el aprendizaje experiencial y, además, afiancen capacidades como la creatividad, el liderazgo y el compromiso con un grupo de interés particular. 
En el año 2016 los centros fueron definidos como Línea de Acción, con el fin de lograr la formación integral de los estudiantes, en cuanto están

constituidos por un grupo de personas de la comunidad universitaria que se han organizado autónomamente alrededor de intereses comunes, una temática o experiencia significativa, a partir de las cuales los jóvenes encuentran elementos en la producción de sentidos para su vida y la construcción de identidad y cultura universitaria. (Universidad de La Salle, 2016, p. 39)

La forma en que se vinculan a estos clubes varía: unos lo hacen por invitación de amigos, mientras que otros lo hacen por iniciativa propia; se acercan al grupo y solicitan el ingreso. Pero todos se integran por el deseo de ampliar sus conocimientos, trabajar por una especie y conocer el medio. Como lo expresó en la entrevista Diego Piragauta, expresidente del Club Bovino: "Lo inicial era activar el club en ferias y darlo a conocer más aquí en la universidad, ya que nos dimos cuenta de que la parte de grandes estaba completamente olvidada y queríamos que el campus volviera a mirar hacia la especie bovina”.

Los estudiantes que han conformado los clubes, hoy Centros de Interés, han respondido en su accionar a

didácticas orientadas a crear espacios adecuados para el acuerdo de normas y valores que favorezcan la convivencia, la tolerancia y la construcción de una ciudadanía democrática. En este sentido, que generen las condiciones adecuadas y suficientes para participar activamente en procesos de transformación política y social, así como para proponer y desarrollar experiencias vinculadas al compromiso con la justicia y la equidad. (Universidad de La Salle, 2008, p. 20)

\section{Líderes en acción: Agroexpo como escenario de preparación de los jóvenes universitarios como futuros constructores de paz en la ruralidad}

Agroexpo es un evento ferial organizado por Corferias que se realiza desde 1977 y que hoy en día es considerado como una de las exposiciones especializadas más importantes de Colombia, Centroamérica y el Caribe, así como una de las más representativas en Latinoamérica, que reúne la más variada y completa muestra agrícola y del sector pecuario nacional e internacional, así como de insumos y cadenas productivas para el procesamiento de alimentos. Es el espacio propicio para el intercambio y actualización tecnológica, por cuanto es un evento netamente agroindustrial y especializado para desarrollar el espíritu empresarial de Colombia hacia el mundo (Agroexpo, 2017).

La Universidad de La Salle, con su programa de Medicina Veterinaria, empezó a participar en esta exposición desde 1992 con un estand promocional y con el apoyo en la logística de toda la feria, que incluye desde el ingreso de los animales, provenientes de diferentes regiones del país (figura 1), su ubicación en los pabellones del recinto ferial, la 
organización y el desarrollo de cada una de las competencias de exhibición del ganado (incluyendo bovinos, ovinos, caprinos y equinos), el cuidado sanitario (figura 2) y, al cierre, la salida del ganado.

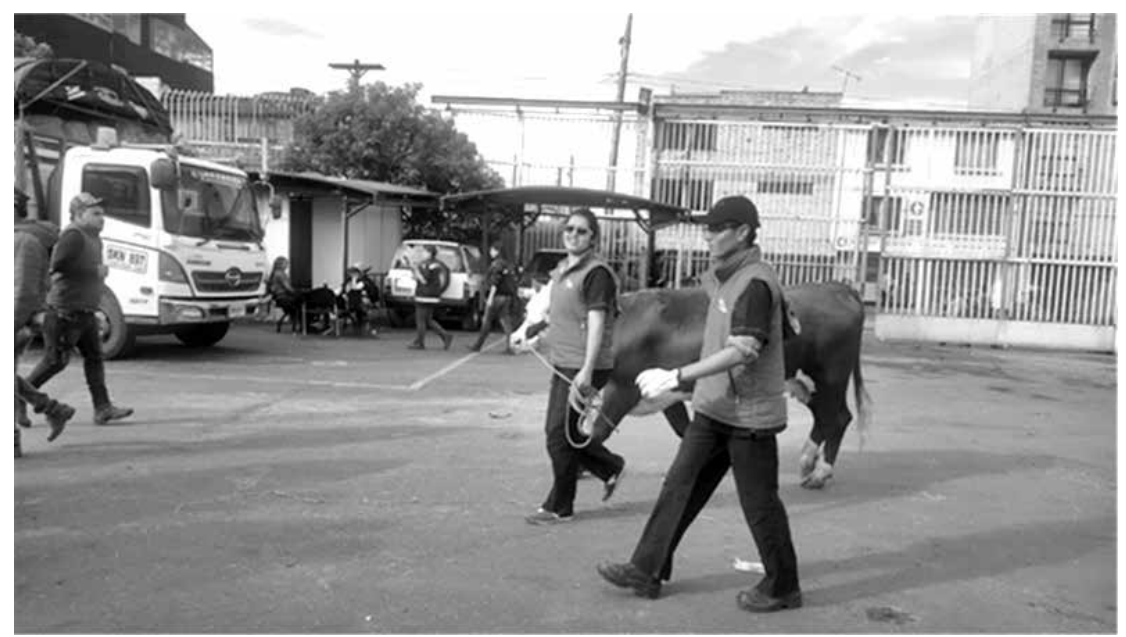

Figura 1. Recepción de animales durante Agroexpo 2017.

Fuente: fotografía tomada por las autoras con consentimiento informado.

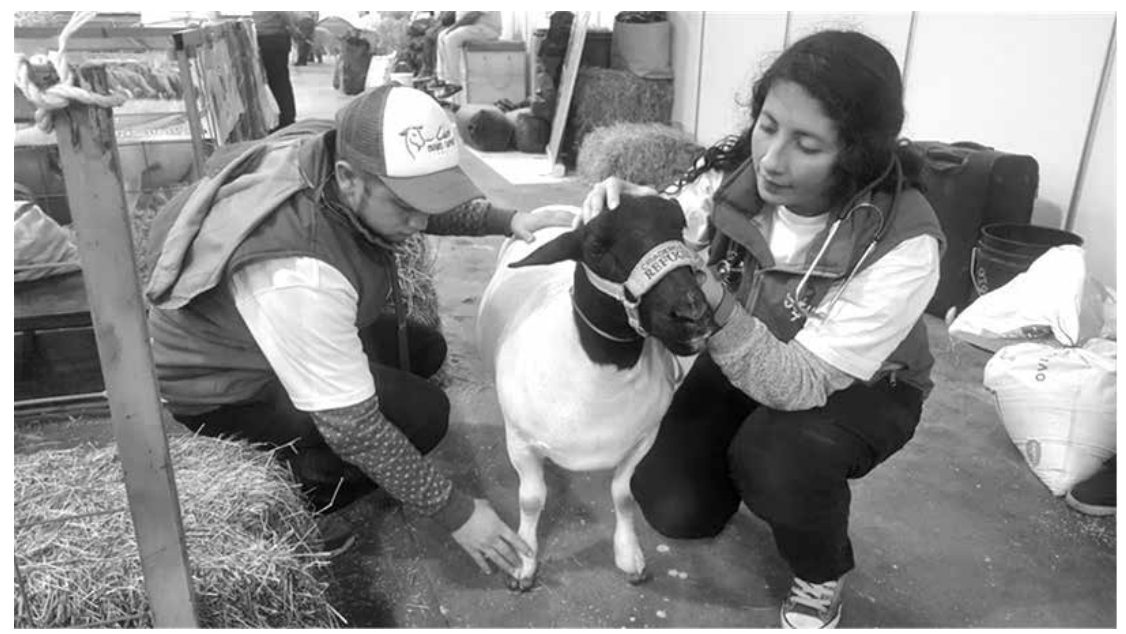

Figura 2. Manejo y cuidado sanitario de los ovinos por estudiantes de La Salle. Fuente: fotografía tomada por las autoras con consentimiento informado.

De esta manera, Agroexpo se ha constituido en un escenario que propicia en los estudiantes "las ganas de trabajar, el conocimiento de las especies, las habilidades en cuanto 
a relaciones con las asociaciones y los diferentes criaderos, la habilidad para cumplir objetivos y misiones a corto plazo", afirma Eric Holguín, presidente del Club Ovino-Caprino.

Todas estas actividades implican una interacción con propietarios, transportadores, estableros y cuidadores en general de estos animales, así como con las diferentes asociaciones de ganaderos y con el público en general. Es una actividad que implica horas largas de trabajo, esfuerzo físico y mental continuo, conflictos por resolver, tareas por realizar, en fin, un sinnúmero de retos que se constituyen como un escenario rápido e intensivo de formación por el trabajo en equipo, por las relaciones con las asociaciones y las personas en general del sector agropecuario y por la puesta en práctica de conocimientos adquiridos en las aulas.

En el marco de la participación de los jóvenes pertenecientes a los clubes en la feria Agroexpo 2017, se realizaron entrevistas semiestructuradas a seis estudiantes de la Facultad de Ciencias Agropecuarias que han sido presidentes o vicepresidentes de los diferentes clubes estudiantiles y que han tenido experiencia en eventos del sector agropecuario. A estas entrevistas se les realizó el respectivo análisis del discurso.

Asimismo, se aplicó una encuesta de percepción a 96 estudiantes que participaron en la logística de Agroexpo 2017, vía electrónica, con preguntas concretas sobre su desempeño y sobre aspectos positivos y negativos de su participación en la exposición. Los resultados de la encuesta evidencian que el 93,6 \% considera que su participación fue importante en su formación profesional y personal. Entre los aspectos que destacan los encuestados se encuentran los siguientes:

"El trato no solo con animales, sino también con personas de otras culturas y ambientes, permitió mi crecimiento personal ayudando a generar nuevas relaciones tanto profesionales como personales". "El liderazgo y el trabajo con todos los muchachos me ayudan a crecer como líder y como persona”. "Me ayudó a ser más paciente, respetuosa, tolerante y a entender que no todos somos iguales". "Tuve la oportunidad de cumplir una función en la que de mi buen desempeño dependía también el buen funcionamiento y desarrollo de la universidad en la feria, teniendo en cuenta la organización de estudiantes, alimentación, turnos, etc.”.

En general, se trata de una experiencia que propicia una mejor apropiación de los conocimientos adquiridos en el aula, así como de las especialidades de la carrera; en tal sentido, para la participación en Agroexpo "se requiere más que nada el compromiso, las ganas de querer hacer las cosas, habilidades fundamentales como saber manejar los animales, conocer sobre razas y, sobre todo, la disposición para querer trabajar con otras personas", dice Diego Piragauta, expresidente del Club Bovino, quien agrega: "Unas habilidades las tenía y otras las fui complementando".

El trabajo durante Agroexpo permitió que los estudiantes tuvieran la oportunidad de responsabilizarse por una serie de actividades que implican el manejo adecuado del relacionamiento, la capacidad de comunicarse asertivamente, solucionar conflictos e interactuar adecuadamente con personas de diferentes niveles educativos y socioeconómicos. 
Es también un escenario que afianza las habilidades para la solución de problemas, de comunicación, relacionamiento, organización y liderazgo. Así lo señala Sebastián Rodríguez, expresidente del Club Equino: "Se requiere la comunicación, es saber decir las cosas de forma políticamente correcta, pues todas las personas que participan lo hacen de manera voluntaria, así que es importante buscar la forma de incentivarlos a que cumplan las funciones. Estas habilidades ya las tenía, pero las reforcé aún más".

Adicionalmente, la participación en Agroexpo genera opciones para que los estudiantes creen relaciones profesionales a futuro, al servir como un escenario de interacción de estos jóvenes con los líderes de los distintos gremios, y así romper con los estereotipos de superioridad que muchas veces limitan o hacen engorrosa la interacción de jóvenes profesionales con los líderes de asociaciones nacionales: "El contacto directo con las asociaciones y el reconocimiento del trabajo es muy importante, ya que voy entrando en un medio en el cual van a conocer mi trabajo aun sin ser egresada", asevera Lorena Pineda, presidenta del Club Bovino.

A la vez, la Feria ha favorecido el fortalecimiento de los centros de interés, tal y como señala Nidia Milena González, vicepresidenta del Club Ovino-Caprino: "Durante Agroexpo hice bastantes contactos para el Club, para sus prácticas y charlas, y para mí es posible hacer prácticas o pasantías". Estos son los escenarios que propician la autonomía como profesionales y el compromiso que necesitan como líderes de un proyecto colectivo, como son los mismos clubes o centros de interés.

Se debe destacar que los jóvenes participantes deben afianzar sus habilidades de dirección y liderazgo, toda vez que bajo su cuidado se encuentra no solo la mayor responsabilidad en el manejo de los animales, sino, más importante aún, la interacción con diferentes actores, como los empresarios que prestan sus animales para la exposición, los funcionarios de la feria, los directivos de las diferentes asociaciones, los encargados del manejo de los animales (palafreneros y estableros) y los conductores encargados del transporte de los animales.

Estos cinco niveles de interrelación marcan de entrada una diferencia en la formación de estos jóvenes, ya que logran poner en práctica sus habilidades de liderazgo fuera del aula y a través del ejercicio como líderes, a la comprensión de los lenguajes, dinámicas y códigos de conducta propios de cada una de estas poblaciones.

Ahora bien, en términos de acercamiento a la ruralidad, debe destacarse la interacción con quienes tienen un contacto directo con las comunidades rurales, y que, en buena parte, pertenecen a estas. Se debe señalar, a propósito, que forman parte de la ruralidad todos aquellos actores que se encuentran inmersos en tal entorno, es decir, tanto empresarios como productores conforman un entramado de relaciones que propician una comprensión propia del mundo, en la configuración de lo que se entiende como ruralidad o mundo rural.

La comprensión del territorio y de las dinámicas que se entretejen en este forma parte de una capacidad social para intervenirlo. Así, al aproximarse al territorio como una 
construcción social, son aquellos líderes capaces de dialogar con los distintos actores sociales que habitan el territorio, quienes pueden a su vez propiciar allí relaciones democráticas e incluyentes.

En este aspecto vuelve a tomar vigencia la comprensión del buen vivir o vivir bien como elemento determinante en el ejercicio del liderazgo rural para la paz. En la medida en que se afiance la capacidad de interacción de estos jóvenes como líderes rurales con los distintos actores del mundo rural, se hace posible una comprensión más cercana a la realidad de este contexto y, por tanto, se propicia un mejor acompańamiento como profesionales a las necesidades de sus interlocutores.

Resulta interesante la manera como la participación en Agroexpo ha propiciado en los jóvenes la toma de conciencia frente a su papel como líderes en el sector rural, lo cual se evidencia en la descripción que realizan de sus aprendizajes. A continuación, se presentan algunos fragmentos de las entrevistas que soportan esta afirmación:

"Se puso en práctica lo visto en clase". "Aprendí manejo animal y establecí vínculos con los gremios". "Porque se conoce más el medio que como profesionales estaremos afrontando". "Porque aprendí más cosas sobre manejo animal, los diferentes puntos de vista de algunos clínicos y sus formas de tratamiento o atención, y también a conocer a los trabajadores de las ganaderías y poder llevar conversaciones donde ninguno es más grande que el otro". (Encuesta a estudiantes colaboradores Agroexpo, 2017)

En efecto, al comparar los estilos de aprendizaje de distintos grupos de edad, García y Rodríguez (2003) concluyen que "con base en el patrón de aprendizaje que presenta el grupo de estudiantes jóvenes, sería recomendable tratar en lo posible de trasladar los contenidos a la práctica y a situaciones de la vida real” (p. 111). En el mismo sentido, los jóvenes destacan la posibilidad de interactuar con distintas personas de distintos niveles en la producción agropecuaria, como una oportunidad de aplicar sus conocimientos y afianzar sus habilidades profesionales.

Esto es evidencia de que "el aprendizaje experiencial ofrece una oportunidad única para conectar la teoría y la práctica. Cuando el alumnado se enfrenta al desafío de responder a un amplio abanico de situaciones reales, se consolida en él un conocimiento significativo, contextualizado, transferible y funcional, y se fomenta su capacidad de aplicar lo aprendido" (Ariza, 2010, p. 90). De manera que Agroexpo y la participación en los centros de interés resultan claramente escenarios propicios para que estos jóvenes afiancen sus habilidades de liderazgo y compromiso social.

\section{Los jóvenes líderes rurales como transformadores de la sociedad siguiendo a San Juan Bautista de La Salle}

La construcción de paz, por otra parte, guarda una estrecha relación con la promoción de liderazgos transformadores y conscientes de la injusticia social que ha sumido al 
sector rural en una condición de inferioridad frente al urbano. En esa medida, se requieren jóvenes capaces de indignarse y de comprometerse con la promoción de una mejor vida para las comunidades.

Galtung (2001) propone comprender la violencia a partir de tres tipos distintos. Así, define la violencia estructural, que está dada por las relaciones inequitativas y de exclusión que existen en la sociedad; la violencia cultural, en la cual, a través de distintas representaciones, discursos y acciones, se justifica la existencia de desigualdad y de la violencia misma en la sociedad, en particular frente a grupos tradicionalmente excluidos. Estas dos categorías son la base para la emergencia de la violencia directa (asesinatos, despojos, riñas, lesiones, etc.), la cual es perceptible en las sociedades y paliada por los Estados, sin que se afecte así sus causas (la violencia estructural y la violencia cultural).

El sector rural en Latinoamérica ha estado sometido de manera recurrente a los tres tipos de violencia, con una mayor intensidad que el sector urbano, en parte debido a las condiciones de inequidad estructural y a la existencia de una sociedad patriarcal que se encarga de justificar tales relaciones inequitativas. Por lo tanto, es necesario volver la mirada al sector rural y propiciar el trabajo conjunto de los profesionales con las comunidades rurales.

"El futuro depende de las nuevas generaciones que lleguen al campo, que exploren y que sean capaces de cultivar esos frutos que, de mano de los profesionales, sigan en crecimiento y sea más valorado en un ámbito sociopolítico". (Lorena Pineda, presidenta del Club Bovino)

En este sentido, los jóvenes profesionales que se aproximan a la realidad del campo colombiano desempeñan un papel fundamental en la modificación de las condiciones que detonan en violencia estructural y cultural, ambas, a través de su ejercicio profesional en el marco del EFL.

Este es un enfoque que hace referencia a la constante interacción entre individuos igualmente dignos, los cuales contribuyen, a través de la deliberación, a una comprensión del mundo más cercana a la realidad, al tiempo que a la formación de capacidades en todos los individuos, en particular en aquellos que se encuentran en una posición desfavorable. (Otálora-Buitrago, 2014. p. 29)

En este sentido son fundamentales dos aspectos: en primer lugar, la lucha contra la violencia cultural, en términos de establecer pautas de interrelación que conduzcan al trato igualitario, digno y horizontal con los distintos actores de las comunidades rurales, con lo cual se romperían las representaciones sociales que les otorgan un halo de superioridad a los profesionales que se acercan del sector urbano al rural.

En segundo lugar, la lucha contra la violencia estructural, al asumir como un compromiso personal y como parte de su proyecto de vida la generación de soluciones innovadoras, creativas y sostenibles en la promoción de una mejor forma de vida o buen vivir en las comunidades rurales. 
A lo largo de este artículo se ha sostenido que los jóvenes profesionales, egresados de la Facultad de Ciencias Agropecuarias, tienen la oportunidad de ejercer como líderes en el sector rural, esto como resultado de su ejercicio profesional, pero también de la formación complementaria que han recibido, fruto de su participación en los clubes equino, bovino, canino y ovino-caprino, hoy denominados centros de interés.

Ahora bien, es este uno de tantos escenarios de formación complementaria que bajo el EFL promueven el compromiso de los jóvenes con la comunidad y que los transforman en agentes del cambio social a través de "didácticas que entusiasman al estudiante con el pensar, el decidir y el servir: pensar con rigurosidad, sentido crítico y creatividad; decidir con responsabilidad, coherencia y oportunidad; servir con solidaridad, valentía y esperanza” (Universidad de La Salle, 2008, pp. 20-21). Al indagar por la percepción que tienen los líderes de los clubes sobre el futuro del sector rural, se encuentra:

"Es un futuro bueno, pero desde que sea bien manejado porque esto está muy regido por el tema político y siempre es complicado el manejo del sector, pero si es bien manejado, es muy bueno y si hay más oferta de profesionales, mucho mejor". (Nidia Milena González, vicepresidenta del Club Ovino-Caprino)

Estos jóvenes desde ya son líderes conscientes de su responsabilidad con el sector rural y expectantes del aporte que harán al país; su apreciación sobre el desarrollo del sector agropecuario es informada y ha sido alimentada por sus vivencias en los clubes y en la feria, en contraste con muchos de los estudiantes que no han tenido contacto con especie alguna ni con el sector en general.

"Considero que el sector agropecuario ha venido creciendo de acuerdo con el último censo, al número de animales, al aporte a los mismos [...] en futuros ańos si se sigue así, va a tener un aporte oportuno, eficiente para futuras generaciones. Es importante el aporte de las universidades agropecuarias que incentiven a trabajar en el sector y esto da a entender que la exigencia a futuro va a ser más de la que hay hoy en día y por tanto debemos aprender, mejorar y actuar en consecuencia”. (Eric Holguín, presidente del Club Ovino-Caprino)

Los jóvenes que han visto permeada su formación profesional por la participación en este tipo de escenarios se convierten no solo en receptores del acto educativo, sino principalmente en transformadores del contexto social, pues pasan a ejercer la función de maestros en el ejercicio de su liderazgo en el sector rural. Asimismo, son multiplicadores de la misión propia de los Hermanos Cristianos de La Salle, en cuanto a la "formación de sujetos críticos, capaces de apropiar conocimientos capaces de dignificarlos como seres humanos y como miembros activos de la sociedad" (Otálora-Buitrago, 2014, p. 18), postura que es claramente asumida por estos jóvenes al hablar sobre su futuro profesional:

"Me pienso dedicar a la parte de reproducción bovina, pues a partir de allí, creo, se basa una muy buena economía del país y también se maneja parte de la salud hacia los seres 
humanos. En estos momentos al sector agropecuario le hace falta más empuje por el gobierno nacional, pues lo ha tenido muy olvidado, pero de igual manera el desarrollo del país viene desde el campo. Por esta razón tenemos que volver nuevamente al campo a trabajar con los animales, trabajar con la tierra para brindar una estabilidad económica y alimentaria para el país”. (Diego Piragauta, expresidente del Club Bovino)

Existe una interacción interesante entre este enfoque, la búsqueda de una definición de ruralidad y de lo rural para el campo colombiano, y en la promoción del buen vivir como alternativa para la determinación de escenarios y oportunidades incluyentes en la disminución y solución a la brecha urbano-rural.

$\mathrm{Y}$ es que es precisamente a través del involucramiento de sujetos críticos que se hace posible la reflexión en torno al buen vivir. En otras palabras, el proceso debe iniciar por la educación en los sentimientos, en la posibilidad de identificar, visibilizar, denunciar y actuar en contra de la injusticia social, tanto como en la interacción con otras formas de ver el mundo a partir del diálogo intercultural activo, propositivo e incluyente.

\section{Conclusiones}

La participación de los jóvenes universitarios en educación complementaria, como la ofertada por los centros de interés, es una oportunidad en la formación de líderes, que como en el caso de los clubes equino, bovino, canino y ovino-caprino, pueden multiplicar su acción de transformación social cuando se aproximan al sector rural como parte de su ejercicio profesional.

La intervención de líderes con un perfil democrático en sectores tradicionalmente excluidos, como el rural, es una alternativa para generar diálogos interculturales, útiles y necesarios en la construcción de un concepto de ruralidad incluyente, más próximo a las expectativas, necesidades y cosmovisiones de los ciudadanos rurales.

Así, son dos las formas en que los jóvenes egresados de la Facultad de Ciencias Agropecuarias pueden contribuir a la construcción de paz: primero, a través de la promoción de soluciones que desde su experticia profesional contribuyan a la disminución de las serias inequidades estructurales que afectan el campo colombiano, $y$, segundo, al actuar como líderes desde el ejercicio mismo de la democracia, es decir, estableciendo relaciones de respeto, cooperación y corresponsabilidad frente a los actores rurales con quienes intervienen durante su ejercicio profesional.

Esta lógica, por supuesto, puede aplicarse también a los jóvenes profesionales de las mismas áreas que deciden desarrollar su ejercicio profesional en las grandes urbes, o bien, a profesionales de otras áreas, quienes aportan soluciones a la sociedad desde la ingeniería, la educación, la salud humana o las ciencias sociales. La ventana de oportunidad que se presenta ante los jóvenes líderes rurales radica en las profundas desigualdades que durante generaciones han perpetuado una brecha urbano-rural, y en la enorme incidencia que fruto de tal inequidad puede tener la promoción de diálogos interculturales en el ejercicio del liderazgo transformacional. 
En el trabajo de planeación para participar en Agroexpo se hizo mucho énfasis en la importancia de realizar una labor muy organizada, con excelentes resultados, que fuera aporte al crecimiento personal, profesional y de relacionamiento para cada individuo. Este reto fue asumido por todos los estudiantes y la gran mayoría de docentes y se vio reflejado en el día a día, lo cual dejó en alto el nombre de la Universidad de La Salle.

Se fortalece así la idea de transformación de la realidad social y el objetivo de ofrecer oportunidades a quienes han sido excluidos de los centros de poder promovida por el ideario de San Juan Bautista de La Salle. Los líderes rurales deben propender por la generación de opciones equitativas y creativas en el campo, y los líderes rurales que se están formando a través de los centros de interés señalados se están empoderando con las herramientas requeridas para lograr la transformación social.

En efecto, el mundo necesita más lasallistas, y los jóvenes líderes de los clubes equino, bovino, canino y ovino-caprino, son un buen ejemplo de ello.

\section{Agradecimientos}

Las autoras agradecen a los estudiantes lasallistas de la Facultad de Ciencias Agropecuarias de la Universidad de La Salle, en Bogotá, por otorgarnos consentimiento informado para publicar los resultados de investigación divulgados en el presente artículo.

\section{Declaración de divullgación}

Las autoras declaran que no existe ningún potencial conflicto de interés relacionado con el artículo.

\section{Financiamiento}

El presente artículo es un producto adicional del "Estudio Longitudinal de Vida 2015-2020" financiado por la Universidad de La Salle, Bogotá, D. C.

\section{Sobre las autoras}

Claudia Aixa Mutis Barreto es médica veterinaria y MSc en Docencia.

Adriana Otálora-Buitrago es economista y politóloga. PhD en Estudios Políticos y MSc en Educación y Desarrollo Humano.

\section{Referencias}

Acosta, A. (2010). El buen vivir, una utopía por (re)construir. En J. Sempere, A. Acosta, S. Abdallah \& M. Ortí, Enfoques sobre bienestar y buen vivir (pp. 11-28). Madrid: CIP-Ecosocial.

Agroexpo. (2017). Sobre la feria [información en página de internet]. Recuperado de https://agroexpo. com/?d=sub\&s=1770\&p=11404\&i=1 
Ariza, M. R. (2010). El aprendizaje experiencial y las nuevas demandas formativas. Antropología Experimental, 8, 89-102.

Burgos Ayala, A. (2016). Buen vivir con la naturaleza en las instituciones educativas: una necesidad en Boyacá, Colombia. Culturales, 4 (2), 185-208.

Caudillo-Félix, G. A. (2012). El buen vivir: un diálogo intercultural. Ra-Ximhai, 8 (2), 345-364.

Galtung, J. (2001). After violence, reconstruction, reconciliation, and resolution. En M. Abu-Nimer (Ed.), Reconciliation, justice and coexistence: Theory and practice (pp. 3-23). Lanham, Maryland: Lexington Books.

García, V., \& Rodríguez, J. (2003). Estilos de aprendizaje y grupos de edad: comparación de dos muestras de estudiantes jóvenes y mayores. Aula Abierta, 82, 97-114.

Mouffe, C. (1999). El retorno de lo político: comunidad, ciudadanía, pluralismo, democracia radical. Paidós Ibérica.

Otálora-Buitrago, A. (2014). Interacción, reconocimiento y dignidad: reflexiones en torno a las pedagogías para la paz a partir del Enfoque Formativo Lasallista. Revista de la Universidad de La Salle, 65, 17-31.

Rodríguez Wallenius, C. (2014). Relaciones campo-ciudad y la construcción de alternativas al desarrollo en Latinoamérica. En F. Hidalgo, F. Houtart \& P. Lizárraga (Eds.), Agriculturas campesinas en Latinoamérica: propuestas y desafíos (pp. 199-215). Quito: IAEN.

Universidad de La Salle. (2008). Enfoque Formativo LaSallista. Bogotá, D. C.: Ediciones Unisalle.

Universidad de La Salle. (2016). Nodos 4. FICU. Formación integral y cultura. Bogotá, D. C.: Ediciones Unisalle. 